\title{
Compact Sobolev embedding theorems involving symmetry and its application
}

\author{
Juanjuan Gao, Peihao Zhao and Yong Zhang
}

\begin{abstract}
Given a bounded regular domain with cylindrical symmetry, functions having such symmetry and belonging to $W^{1, p}$ can be embedded compactly into some weighted $L^{q}$ spaces, with $q$ superior to the critical Sobolev exponent. A similar result is also obtained for variable exponent Sobolev space $W^{1, p(x)}$. Furthermore, we give a simple application to the $p(x)$-Laplacian problem.
\end{abstract}

AMS Subject Classification (2000). 35D05, 35J60, 46E30, 46E35.

Keywords. Sobolev embedding, Cylindrical symmetry, $p(x)$-Laplacian, Weak solution.

\section{Introduction}

Let $\Omega$ be a bounded domain in $\mathbb{R}^{N}$ with cone property. Sobolev embedding theorems state that any $L^{p}$ functions with $L^{p}$ derivatives belong to the space $L^{q}$ with $q \in\left(1, p^{*}\right]$, where $1<p<N, p^{*}=\frac{N p}{N-p}$. In fact, there is a continuous embedding from $W^{1, p}(\Omega)$ into $L^{q}(\Omega)$ for any $q \in\left(1, p^{*}\right]$. Furthermore, when $q<p^{*}$, the embedding mapping is compact. Due to the compactness, we can solve differential equations by variational arguments.

Many authors studied the solvability of elliptic problems on the domain with special geometric and topological properties. Generally speaking, some kinds of the geometric and topological properties of the domain lead to the solvability of elliptic problems. We note that symmetry can improve Sobolev embeddings (see [1-4] and references therein). If $\Omega=\mathbb{R}^{N}, \mathrm{P}$. L. Lions (see $[1,2])$ showed that the embedding $W_{r}^{1, p}\left(\mathbb{R}^{N}\right) \hookrightarrow L^{q}\left(\mathbb{R}^{N}\right)$ is compact for any $p<q<p^{*}$, where $W_{r}^{1, p}\left(\mathbb{R}^{N}\right):=\left\{u \in W^{1, p}\left(\mathbb{R}^{N}\right): u(x)=u(|x|), \forall x \in \mathbb{R}^{N}\right\}$. Especially in [4], for a bounded regular domain with cylindrical symmetry, Wang has got a compact embedding from the Hilbert space $W_{0, s}^{1,2}(\Omega)$ to the 
Lebesgue space $L_{h}^{q}(\Omega)$ for any $1<q<2^{*}+\tau$, where $\tau$ is a positive constant, $W_{0, s}^{1,2}(\Omega):=\left\{u \in H_{0}^{1}(\Omega): u\left(x_{1}, x_{2}\right)=u\left(x_{1},\left|x_{2}\right|\right)\right\}, L_{h}^{q}(\Omega):=\left\{u \in L^{q}(\Omega) \mid\right.$ $\left.\int_{\Omega} h|u|^{q} d x<\infty\right\}$. So one can deal with the solvability of elliptic problems with critical or supercritical nonlinearities.

In recent years, there has been an increasing interest in the study of variational problems with variable exponent (see [5-9]). Sobolev embedding theorems for the variable exponent Sobolev space $W^{1, p(x)}(\Omega)$ have been studied by many authors (see e.g. [10-16]). In fact, for $1<p(x)<N$, if $\Omega$ is bounded and $p(x) \in C(\bar{\Omega})$, there is a compact embedding from $W^{1, p(x)}(\Omega)$ to $L^{q(x)}(\Omega)$ for any $q(x)$ satisfying $q(x) \in C(\bar{\Omega})$ and $1<q(x)<p^{*}(x)=\frac{N p(x)}{N-p(x)}$, $\forall x \in \bar{\Omega}$ (see [14]). If $\Omega$ is unbounded, $p(x)$ is Lipschitz continuous, the embed$\operatorname{ding} W^{1, p(x)}(\Omega) \hookrightarrow L^{q(x)}(\Omega)$ is continuous for any measurable function $q(x)$ satisfying $p(x) \leq q(x) \leq p^{*}(x)$ a.e. in $\bar{\Omega}$, but not compact (see [15]). But if $\Omega=$ $\mathbb{R}^{N}, \mathrm{X}$. L. Fan extended the results of Lions and obtained that $W_{r}^{1, p(x)}\left(\mathbb{R}^{N}\right)$ can be embedded compactly into $L^{q(x)}\left(\mathbb{R}^{N}\right)$ if $p(x)$ is radially symmetric and $p(x) \ll q(x) \ll p^{*}(x), \forall x \in \mathbb{R}^{N}$ (see $[16]$ ).

In this paper, we will extend Wang's embedding results (see [4]) from $W^{1,2}(\Omega)$ to $W^{1, p}(\Omega)$ and $W^{1, p(x)}(\Omega)$ respectively, where $\Omega$ is a bounded regular domain with cylindrical symmetry. To be specific, in Sect. 3 we first prove that there is a positive constant $\tau$ such that the embedding mapping $W_{0, s}^{1, p}(\Omega) \hookrightarrow L_{h}^{q}(\Omega)$ is compact for $1<q<p^{*}+\tau$. Next, motivated by the idea in $[14,16]$, we obtain in Sect. 4 that the embedding mapping $W_{0, s}^{1, p(x)}(\Omega) \hookrightarrow$ $L_{h}^{q(x)}(\Omega)$ is compact for $1<q(x)<p^{*}(x)+\tau$. Finally in Sect. 5 , we give a simple application to the $p(x)$-Laplacian problem

$$
\left\{\begin{array}{l}
-\Delta_{p(x)} u \equiv-\operatorname{div}\left(|\nabla u|^{p(x)-2} \nabla u\right)=f(x, u) \quad x \in \Omega \\
u=0 \quad x \in \partial \Omega
\end{array}\right.
$$

where $f(x, t)=\lambda f_{1}(x, t)+h(x) f_{2}(x, t), f_{i}: \Omega \times \mathbb{R} \rightarrow \mathbb{R}$ is a Carathéodory function and satisfies $\left|f_{i}(x, t)\right| \leq C_{1}+C_{2}|t|^{q_{i}(x)-1}, \forall x \in \Omega \times \mathbb{R}, i=1,2 ; \lambda$ is a positive real parameter. Under some other assumptions on $f_{i}$ and $h$, we find two nontrivial weak solutions of $(\mathrm{P})$ for $q_{1}(x)<p(x)<q_{2}(x)<p^{*}(x)+\tau$.

Here, we obtain weak solutions for the case with a little more supercritical growth. Indeed, in [17] the authors also have obtained solutions without the condition $q(x)<p^{*}(x)$, for some $x \in \bar{\Omega}$. Precisely, with the continuity and oddness of $f(x, t)$, utilizing Symmetric Mountain Pass Lemma, they proved that $(\mathrm{P})$ has nontrivial weak solutions if there exists an $x_{0} \in \Omega$ such that $q\left(x_{0}\right)<p\left(x_{0}\right)$ and some other conditions hold near the point $x_{0}$. That is, $q(x)$ can be superior to $p^{*}(x)$ for nonzero measurable subset of $\Omega$.

\section{Preliminaries}

Let $\Omega=\Omega_{1} \times \Omega_{2} \subset \mathbb{R}^{N}$, with $\Omega_{1} \subset \mathbb{R}^{m}(m \geq 1)$ being a bounded regular domain, and $\Omega_{2} \subset \mathbb{R}^{k}(k \geq 2)$ being a ball of radius $\mathrm{R}$, centered at the origin.

We denote

$A, B, C, c$, are positive constants (possibly different); 
$\left(\mathbf{H}_{\mathbf{1}}\right): h(x)$ is a nonnegative Hölder continuous function in $\bar{\Omega}$, radially symmetric with respect to $x_{2} \in \Omega_{2}$, and satisfying $h\left(x_{1}, 0\right)=0$;

$L_{h}^{p}(\Omega)=\left\{\left.u \in L^{p}(\Omega)\left|\int_{\Omega} h\right| u\right|^{p} d x<\infty\right\}$ with the norm $|u|_{h, p}=$ $\left(\int_{\Omega} h|u|^{p} d x\right)^{1 / p}, 1<p<\infty$

$S(\Omega)=\left\{u: u\right.$ is real measurable, $\left.u\left(\cdot, x_{2}\right)=u\left(\cdot,\left|x_{2}\right|\right)\right\} ;$

$W_{0, s}^{1, p}(\Omega):=W_{0}^{1, p}(\Omega) \cap S(\Omega)$, with the norm $\|u\|_{p}=\left(\int_{\Omega}|\nabla u|^{p} d x\right)^{\frac{1}{p}}$.

In order to discuss our embedding theorem in Sect. 4 , we need some theories on the space $W^{1, p(x)}(\Omega)$, which we call variable exponent Sobolev spaces. Firstly, we state some basic properties which will be used later (for details, see $[14,16])$.

Set

$$
C_{+}(\bar{\Omega}):=\{p(x): p(x) \in C(\bar{\Omega}), p(x)>1, \quad \forall x \in \bar{\Omega}\},
$$

and denote

$$
p_{+}(U)=\sup _{x \in U} p(x), \quad p_{-}(U)=\inf _{x \in U} p(x)
$$

for any nonempty subset $U \subseteq \Omega$ and any $p(x) \in C_{+}(\bar{\Omega})$.

We define

$L^{p(x)}(\Omega):=\left\{u: u\right.$ is real measurable, $\left.\int_{\Omega}|u|^{p(x)} d x<\infty\right\}$ with the norm

$$
|u|_{p(x)}=|u|_{L^{p(x)}(\Omega)}:=\inf \left\{\lambda>0: \int_{\Omega}\left|\frac{u}{\lambda}\right|^{p(x)} d x \leq 1\right\} ;
$$

$L_{h}^{p(x)}(\Omega):=\left\{u \in L^{p(x)}(\Omega): \int_{\Omega} h|u|^{p(x)} d x<\infty\right\}$ with the norm

$$
|u|_{h, p(x)}=|u|_{L_{h}^{p(x)}(\Omega)}:=\inf \left\{\lambda>0: \int_{\Omega} h\left|\frac{u}{\lambda}\right|^{p(x)} d x \leq 1\right\} ;
$$

$W^{1, p(x)}(\Omega):=\left\{u \in L^{p(x)}(\Omega) ;|\nabla u| \in L^{p(x)}(\Omega)\right\}$ with the norm

$$
\|u\|_{1, p(x)}=\|u\|_{W^{1, p(x)}(\Omega)}:=|u|_{p(x)}+|\nabla u|_{p(x)} .
$$

Denote $W_{0}^{1, p(x)}(\Omega)$ by the closure of $C_{0}^{\infty}(\Omega)$ in $W^{1, p(x)}(\Omega)$ with respect to the norm $\|\cdot\|_{1, p(x)}$ and $W_{0, s}^{1, p(x)}(\Omega):=W_{0}^{1, p(x)}(\Omega) \cap S(\Omega)$.

Proposition 2.1 (see $[13,14])$. $L^{p(x)}(\Omega), W^{1, p(x)}(\Omega)$ and $W_{0}^{1, p(x)}(\Omega)$ are all separable, reflexive and uniformly convex Banach spaces.

Proposition 2.2 (see [16]). If $u \in L^{p(x)}(\Omega),|u|_{p(x)}=\lambda$, then

(1) $\lambda<1(=1,>1) \Leftrightarrow \int_{\Omega}|u(x)|^{p(x)} d x<1(=1,>1)$,

(2) if $\lambda \geq 1$, then $\lambda^{p_{-}} \leq \int_{\Omega}|u(x)|^{p(x)} d x \leq \lambda^{p_{+}}$,

(3) if $\lambda \leq 1$, then $\lambda^{p_{+}} \leq \int_{\Omega}|u(x)|^{p(x)} d x \leq \lambda^{p_{-}}$,

(4) $\int_{\Omega}\left|u_{n}(x)\right|^{p(x)} d x \rightarrow 0 \Leftrightarrow\left|u_{n}(x)\right|_{p(x)} \rightarrow 0$,

(5) $\int_{\Omega}\left|u_{n}(x)\right|^{p(x)} d x \rightarrow \infty \Leftrightarrow\left|u_{n}(x)\right|_{p(x)} \rightarrow \infty$.

Remark 2.3 (see [6]). Set $\rho(u)=\int_{\Omega} h(x)|u(x)|^{p(x)} d x$. For $u \in L_{h}^{p(x)}(\Omega)$, we have 
(1) $|u|_{h, p(x)}<(=;>) 1 \Leftrightarrow \rho(u)<(=;>) 1$,

(2) If $|u|_{h, p(x)}>1$, then $|u|_{h, p(x)}^{p_{-}} \leq \rho(u) \leq|u|_{h, p(x)}^{p_{+}}$,

(3) If $|u|_{h, p(x)}<1$, then $|u|_{h, p(x)}^{p_{+}} \leq \rho(u) \leq|u|_{h, p(x)}^{p_{-}}$.

Remark 2.4 (see [16]). For $u \in W^{1, p(x)}(\Omega)$, if we define

$$
\left|\|u \mid\|:=\inf \left\{\lambda>0: \int_{\Omega} \frac{|u|^{p(x)}+|\nabla u|^{p(x)}}{\lambda^{p(x)}} d x \leq 1\right\},\right.
$$

then $|\| \cdot|||$ is an equivalent norm on $W^{1, p(x)}(\Omega)$. In fact, we have the inequality $\frac{\|u\|_{1, p(x)}}{2} \leq\left|\|u \mid\| \leq 2\|u\|_{1, p(x)}\right.$. Hence, we have

(1) if $\int_{\Omega}\left(|u|^{p(x)}+|\nabla u|^{p(x)}\right) d x \geq 1$, then

$$
c_{1}\|u\|_{1, p(x)}^{p_{-}} \leq \int_{\Omega}\left(|u|^{p(x)}+|\nabla u|^{p(x)}\right) d x \leq c_{2}\|u\|_{1, p(x)}^{p_{+}},
$$

(2) if $\int_{\Omega}\left(|u|^{p(x)}+|\nabla u|^{p(x)}\right) d x \leq 1$, then

$$
c_{3}\|u\|_{1, p(x)}^{p_{+}} \leq \int_{\Omega}\left(|u|^{p(x)}+|\nabla u|^{p(x)}\right) d x \leq c_{4}\|u\|_{1, p(x)}^{p_{-}},
$$

where $c_{i}, i=1, \ldots, 4$, are independent of $u$.

The propositions in the following are some required embedding theorems, which play important roles in our proof of Theorem 4.1.

Proposition 2.5 (see [14]). Assume that $p_{1}(x), p_{2}(x) \in C_{+}(\bar{\Omega})$. If $p_{1}(x) \leq p_{2}(x)$, $\forall x \in \Omega$, then $L^{p_{2}(x)}(\Omega) \hookrightarrow L^{p_{1}(x)}(\Omega)$ and the embedding is continuous.

Proposition 2.6 (see [14]). If $q(x) \in C_{+}(\bar{\Omega})$ and $q(x)<p^{*}(x), \forall x \in \Omega$, then the embedding from $W^{1, p(x)}(\Omega)$ to $L^{p(x)}(\Omega)$ is compact and continuous, where

$$
p^{*}(x):=\left\{\begin{array}{l}
\frac{N p(x)}{N-p(x)}, \quad \text { if } p(x)<N, \\
\infty, \quad \text { if } \quad p(x) \geq N .
\end{array}\right.
$$

Proposition 2.7 (see [14]). Assume that $p_{1}(x), p_{2}(x) \in C_{+}(\bar{\Omega})$. If $p_{1}(x) \leq p_{2}(x)$, $\forall x \in \Omega$, then $W^{m, p_{2}(x)}(\Omega)$ can be embedded into $W^{m, p_{1}(x)}(\Omega)$ continuously.

\section{Compact embedding for constant exponent Sobolev space}

In this section, we mainly consider the case of $1<p<N$. Now, we show some basic Lemmas first.

Lemma 3.1. Let $\operatorname{dim} \Omega_{2}=k>p$, then for $u \in W_{0, s}^{1, p}(\Omega)$, we have

$$
|u(x)| \leq \frac{C_{k, p}}{\left|x_{2}\right|^{\frac{k-p}{p}}}\left(\int_{\Omega_{2}}\left|\nabla_{2} u\right|^{p}\right)^{\frac{1}{p}}, \quad \forall x \in \Omega,
$$

where $C_{k, p}$ is a constant depending only on $k, p$. 
Proof. Let $u(x) \in W_{0, s}^{1, p}(\Omega) \bigcap C_{0}^{\infty}(\Omega)$. Then

$$
u\left(x_{1}, x_{2}\right)-u\left(x_{1}, R\right)=-\int_{\left|x_{2}\right|}^{R} u_{t}\left(x_{1}, t\right) d t .
$$

By Hölder inequality, we have

$$
\begin{aligned}
|u(x)| & \leq \int_{\left|x_{2}\right|}^{R}\left|u_{t}\left(x_{1}, t\right)\right| d t=\int_{\left|x_{2}\right|}^{R}\left|u_{t}\left(x_{1}, t\right)\right| t^{\frac{k-1}{p}} t^{\frac{1-k}{p}} d t \\
& \leq\left\{\int_{\left|x_{2}\right|}^{R}\left|u_{t}\left(x_{1}, t\right)\right|^{p} t^{k-1} d t\right\}^{\frac{1}{p}}\left\{\int_{\left|x_{2}\right|}^{R} t^{\frac{1-k}{p-1}} d t\right\}^{\frac{p-1}{p}} \\
& =\left\{\frac{1}{\omega_{k-1}} \int_{\left|x_{2}\right|}^{R} \int_{S}\left|u_{t}\left(x_{1}, t\right)\right|^{p} t^{k-1} d s d t\right\}^{\frac{1}{p}}\left(\frac{k-p}{p-1}\right)^{-\frac{p-1}{p}}\left(\left|x_{2}\right|^{-\frac{k-p}{p}}\right) \\
& =\omega_{k-1}^{-\frac{1}{p}}\left(\int_{\Omega_{2}}\left|\nabla_{2} u\right|^{p}\right)^{\frac{1}{p}}\left(\frac{k-p}{p-1}\right)^{-\frac{p-1}{p}}\left(\left|x_{2}\right|^{-\frac{k-p}{p}}\right) \\
& =\frac{C_{k, p}}{\left|x_{2}\right|^{\frac{k-p}{p}}}\left(\int_{\Omega_{2}}\left|\nabla_{2} u\right|^{p}\right)^{\frac{1}{p}},
\end{aligned}
$$

where $\omega_{k-1}$ stands for the area of the unit sphere. So we complete the proof.

Lemma 3.2. Let $\operatorname{dim} \Omega_{2}=k$ and $b>1$ be a real constant, then for $u \in W_{0, s}^{1, p}(\Omega)$ and all $x \in \Omega$, we have

$$
|u| \leq \frac{A_{k, p}}{\left|x_{2}\right|^{k-1}} \int_{\Omega_{2}}|\nabla u|, \quad|u|^{b} \leq \frac{b A_{k, p}}{\left|x_{2}\right|^{k-1}} \int_{\Omega_{2}}|u|^{b-1}|\nabla u| .
$$

Proof. The proof is the same as Lemma 3.1, so we omit the details.

To simplify our later proof of the theorems, we will employ Sobolev's operator and its properties. Given $f \in L^{1}(\Omega)$, define

$$
V(f)\left(x_{1}\right)=\int_{\Omega_{1}} \frac{\left|f\left(y_{1}\right)\right|}{\left|x_{1}-y_{1}\right|^{m-1}} d y_{1} .
$$

Then the operator has the following property and its proof can be found in [18].

Lemma 3.3. Let $0 \leq \delta\left(q_{1}, q_{2}\right)=\frac{1}{q_{1}}-\frac{1}{q_{2}}<\frac{1}{m}$, then the operator $V$ maps $L^{q_{1}}(\Omega)$ continuously into $L^{q_{2}}(\Omega)$ for any $1 \leq q_{2} \leq \infty$ and

$$
\|V(f)\|_{q_{2}} \leq C\|f\|_{q_{1}} .
$$

Lemma 3.4. If $u \in W_{0}^{1,1}(\Omega)$, then

$$
|u(x)| \leq \frac{V(|\nabla u|)}{N \omega_{N}} \quad \text { a.e. in } \Omega,
$$

where $\omega_{N}$ also stands for the area of the unit sphere.

In order to give our main result in this section, we first show the following three theorems. 
Theorem 3.1. Let $\operatorname{dim} \Omega_{1}=m \geq 2$ and $\operatorname{dim} \Omega_{2}=k>p$. Suppose that $h(x)=$ $\left|x_{2}\right|^{l}, l>0$ being a real constant. Then the embedding $W_{0, s}^{1, p}(\Omega) \hookrightarrow L_{h}^{q}(\Omega)$ is compact for $1<q<p^{*}+\frac{p l^{\prime}}{N-p}$, where $0<l^{\prime}<\min \left\{\frac{(k-p) p}{m}, l\right\}$.

Proof. In this section, we only prove the continuity of the embedding, that is $\left(\int_{\Omega}\left|x_{2}\right|^{l}|u|^{q}\right)^{\frac{1}{q}} \leq A\left(\int_{\Omega}|\nabla u|^{p}\right)^{\frac{1}{p}}$. The compactness follows a standard procedure which can be found, for example in [3] for the proof of Kondrachov theorem. Now, let $0<a<p$ and $0<b<p$ be real constants. For $u(x) \in W_{0, s}^{1, p}(\Omega)$, from Lemmas 3.1 and 3.4, we can easily get the following

$$
\left|x_{2}\right|^{l}|u|^{a+b} \leq \frac{B\left|V\left(\nabla_{1} u\right)\right|^{a}}{\left|x_{2}\right|^{\frac{k-p}{p} b-l}}\left(\int_{\Omega_{2}}\left|\nabla_{2} u\right|^{p}\right)^{\frac{b}{p}} .
$$

Integrating on $\Omega_{1}$,

$$
\int_{\Omega_{1}}\left|x_{2}\right|^{l}|u|^{a+b} \leq \frac{B}{\left|x_{2}\right|^{\frac{k-p}{p} b-l}} \int_{\Omega_{1}}\left|V\left(\nabla_{1} u\right)\right|^{a}\left(\int_{\Omega_{2}}\left|\nabla_{2} u\right|^{p}\right)^{\frac{b}{p}} .
$$

By Hölder inequality, we get

$$
\int_{\Omega_{1}}\left|x_{2}\right|^{l}|u|^{a+b} \leq \frac{B}{\left|x_{2}\right|^{\frac{k-p}{p} b-l}}\left(\int_{\Omega_{1}} \mid V\left(\nabla_{1} u\right)^{\frac{p a}{p-b}}\right)^{\frac{p-b}{p}}\left(\int_{\Omega}\left|\nabla_{2} u\right|^{p}\right)^{\frac{b}{p}} .
$$

Choosing $q_{2}=\frac{p a}{p-b}$ in Lemma 3.3, if $q_{2}>1$ and $\frac{1}{p}-\frac{p a}{p-b}<\frac{1}{m}$, then

$$
\int_{\Omega_{1}}\left|x_{2}\right|^{l}|u|^{a+b} \leq \frac{B}{\left|x_{2}\right|^{\frac{k-p}{p} b-l}}\left(\int_{\Omega_{1}}\left|\nabla_{1} u\right|^{p}\right)^{\frac{a}{p}}\left(\int_{\Omega}\left|\nabla_{2} u\right|^{p}\right)^{\frac{b}{p}} .
$$

Integrating on $\Omega_{2}$,

$$
\int_{\Omega}\left|x_{2}\right|^{l}|u|^{a+b} \leq \int_{\Omega_{2}} \frac{B}{\left|x_{2}\right|^{\frac{k-p}{p} b-l}}\left(\int_{\Omega_{1}}\left|\nabla_{1} u\right|^{p}\right)^{\frac{a}{p}}\left(\int_{\Omega}\left|\nabla_{2} u\right|^{p}\right)^{\frac{b}{p}} .
$$

From Hölder inequality, and let $\left(\frac{k-p}{p} b-l\right) \frac{p}{p-a}<k$, we have

$$
\int_{\Omega}\left|x_{2}\right|^{l}|u|^{a+b} \leq\left(\int_{\Omega_{2}} \frac{B}{\left|x_{2}\right|^{\left(\frac{k-p}{p} b-l\right) \frac{p}{p-a}}}\right)^{\frac{p-a}{p}}\left(\int_{\Omega}|\nabla u|^{p}\right)^{\frac{b+a}{p}} .
$$

Notice that if $\left(\frac{k-p}{p} b-l\right) \frac{p}{p-a}<k$, then

$$
\int_{\Omega}\left|x_{2}\right|^{l}|u|^{a+b} \leq C\left(\int_{\Omega}|\nabla u|^{p}\right)^{\frac{b+a}{p}} .
$$

Next we solve the following inequalities

$$
\left\{\begin{array}{l}
\left(\frac{k-p}{p} b-l\right) \frac{p}{p-a}<k, \\
\frac{1}{p}-\frac{p a}{p-b}<\frac{1}{m} \\
\frac{p a}{p-b}>1 .
\end{array}\right.
$$


Taking $a=\frac{m p+m l^{\prime}}{N-p}$ and $b=\frac{p k+p l^{\prime}-l^{\prime} m-\epsilon}{N-p}$, where $\epsilon$ is small enough and $0<$ $l^{\prime}<\min \left\{\frac{(k-p) p}{m}, l\right\}$. By elementary calculation, we know that $a$ and $b$ satisfy the inequalities (3.1) and $a+b>p^{*}$. So we complete the proof.

Theorem 3.2. Let $\operatorname{dim} \Omega_{1}=m \neq 1$ and $1<\operatorname{dim} \Omega_{2}=k \leq p$. Suppose that $h(x)=\left|x_{2}\right|^{l}, l>0$ being a real constant. Then the embedding $W_{0, s}^{1, p}(\Omega) \hookrightarrow$ $L_{h}^{q}(\Omega)$ is compact for $1<q<p^{*}+\frac{p l^{\prime}}{N-p}$, where $0<l^{\prime}<\min \left\{\frac{k-1}{m}, l\right\}$.

Proof. Taking $\alpha, \beta \in(0,1)$ and $b>1$ a positive constant. Let $u(x) \in W_{0, s}^{1, p}(\Omega)$. By Lemmas 3.2 and 3.4 we can easily get

$$
\left|x_{2}\right|^{l}|u|^{b(\alpha+\beta)} \leq \frac{B\left[V\left(|u|^{b-1} \nabla_{1} u\right)\right]^{\alpha}}{\left|x_{2}\right|^{(k-1) \beta-l}}\left(\int_{\Omega_{2}}|u|^{b-1}|\nabla u|\right)^{\beta} .
$$

Integrating on $\Omega_{1}$,

$$
\int_{\Omega_{1}}\left|x_{2}\right|^{l}|u|^{b(\alpha+\beta)} \leq \frac{B}{\left|x_{2}\right|^{(k-1) \beta-l}} \int_{\Omega_{1}}\left[V\left(|u|^{b-1} \nabla_{1} u\right)\right]^{\alpha}\left(\int_{\Omega_{2}}|u|^{b-1}|\nabla u|\right)^{\beta} .
$$

According to Hölder inequality,

$\int_{\Omega_{1}}\left|x_{2}\right|^{l}|u|^{b(\alpha+\beta)} \leq \frac{B}{\left|x_{2}\right|^{(k-1) \beta-l}}\left(\int_{\Omega_{1}}\left[V\left(|u|^{b-1} \nabla_{1} u\right)\right]^{\frac{\alpha}{1-\beta}}\right)^{1-\beta}\left(\int_{\Omega}|u|^{b-1}|\nabla u|\right)^{\beta}$.

By Lemma 3.3, if $1<\frac{\alpha}{1-\beta}<\frac{m}{m-1}$, then

$$
\int_{\Omega_{1}}\left[V\left(|u|^{b-1} \nabla_{1} u\right)\right]^{\frac{\alpha}{1-\beta}} \leq\left(\int_{\Omega_{1}}|u|^{b-1}\left|\nabla_{1} u\right|\right)^{\frac{\alpha}{1-\beta}} .
$$

So,

$$
\int_{\Omega_{1}}\left|x_{2}\right|^{l}|u|^{b(\alpha+\beta)} \leq \frac{B}{\left|x_{2}\right|^{(k-1) \beta-l}}\left(\int_{\Omega_{1}}|u|^{b-1}\left|\nabla_{1} u\right|\right)^{\alpha}\left(\int_{\Omega}|u|^{b-1}|\nabla u|\right)^{\beta} .
$$

Integrating on $\Omega_{2}$, we can get

$$
\int_{\Omega}\left|x_{2}\right|^{l}|u|^{b(\alpha+\beta)} \leq \int_{\Omega_{2}} \frac{B}{\left|x_{2}\right|^{(k-1) \beta-l}}\left(\int_{\Omega_{1}}|u|^{b-1}\left|\nabla_{1} u\right|\right)^{\alpha}\left(\int_{\Omega}|u|^{b-1}|\nabla u|\right)^{\beta} .
$$

Using the Hölder inequality again,

$$
\int_{\Omega}\left|x_{2}\right|^{l}|u|^{b(\alpha+\beta)} \leq B\left(\int_{\Omega_{2}} \frac{1}{\left|x_{2}\right|^{\frac{(k-1) \beta-l}{1-\alpha}}}\right)^{1-\alpha}\left(\int_{\Omega}|u|^{b-1}|\nabla u|\right)^{\alpha+\beta} .
$$

If $\frac{(k-1) \beta-l}{1-\alpha}<k=\operatorname{dim}\left(\Omega_{2}\right)$, then the first term of the right hand of $(3.2)$ is integrable. So,

$$
\int_{\Omega}\left|x_{2}\right|^{l}|u|^{b(\alpha+\beta)} \leq B\left(\int_{\Omega}|u|^{b-1}|\nabla u|\right)^{\alpha+\beta} .
$$

Applying the Hölder inequality to the right hand of (3.3), we have

$$
\int_{\Omega}\left|x_{2}\right|^{l}|u|^{b(\alpha+\beta)} \leq B\left(\int_{\Omega}|u|^{(b-1) \frac{p}{p-1}}\right)^{\frac{(\alpha+\beta)(p-1)}{p}}\left(\int_{\Omega}|\nabla u|^{p}\right)^{\frac{\alpha+\beta}{p}} .
$$


Notice that if $1 \leq(b-1) \frac{p}{p-1} \leq p^{*}$, the Sobolev space $W_{0}^{1, p}$ can be embedded into $L^{(b-1) \frac{p}{p-1}}$. So

$$
\int_{\Omega}\left|x_{2}\right|^{l}|u|^{b(\alpha+\beta)} \leq B\left(\int_{\Omega}|\nabla u|^{p}\right)^{\frac{b(\alpha+\beta)}{p}} .
$$

Next we solve the following inequalities

$$
\left\{\begin{array}{l}
\frac{(k-1) \beta-l}{1-\alpha}<k, \\
b \geq \frac{2 p-1}{p}, \\
b \leq \frac{N p-p}{N-p}, \\
1<\frac{\alpha}{1-\beta}<\frac{m}{m-1} .
\end{array}\right.
$$

Now taking $b=\frac{N p-p}{N-p}, \alpha=\frac{m\left(1+l^{\prime}\right)-\eta}{N-1}$, where $\eta$ is small enough, $\beta=\frac{k-(m-1) l^{\prime}}{N-1}$. By elementary calculation and taking $0<l^{\prime}<\min \left\{\frac{k-1}{m}, l\right\}$, we can get that $b, \alpha$ and $\beta$ satisfy the inequalities (3.4) and $b(\alpha+\beta)>p^{*}$. This completes the proof.

In the above two theorems, we make the assumption that $m \neq 1$. If $m=1$, we have the following theorem.

Theorem 3.3. Let $\operatorname{dim} \Omega_{1}=m=1$ and $\operatorname{dim} \Omega_{2}=k \geq 2$. Suppose that $h(x)=$ $\left|x_{2}\right|^{l}, l>0$ being a real constant. Then the embedding $W_{0, s}^{1, p}(\Omega) \hookrightarrow L_{h}^{q}(\Omega)$ is compact for $1<q<p^{*}+\frac{p l^{\prime}}{1+k-p}$, where $0<l^{\prime}<l$.

Proof. Let $b>1$ and $0<c<1$ be real numbers. For $u \in W_{0, s}^{1, p}(\Omega)$, we draw from Lemmas 3.2 and 3.4 the following

$$
\left|x_{2}\right|^{l}|u|^{b(1+c)} \leq \frac{B}{\left|x_{2}\right|^{k-1-l}}\left(\int_{\Omega_{1}}|u|^{b-1}|\nabla u|\right)^{c} \int_{\Omega_{2}}|u|^{b-1}|\nabla u| .
$$

Integrating on $\Omega_{1}$, we have

$$
\int_{\Omega_{1}}\left|x_{2}\right|^{l}|u|^{b(1+c)} \leq \frac{B}{\left|x_{2}\right|^{k-1-l}}\left(\int_{\Omega_{1}}|u|^{b-1}|\nabla u|\right)^{c} \int_{\Omega}|u|^{b-1}|\nabla u| .
$$

Then integrating on $\Omega_{2}$ and using the Hölder inequality,

$$
\int_{\Omega}\left|x_{2}\right|^{l}|u|^{b(1+c)} \leq B\left(\int_{\Omega_{2}} \frac{1}{\left|x_{2}\right|^{\frac{k-1-l}{1-c}}}\right)^{1-c}\left(\int_{\Omega}|u|^{b-1}|\nabla u|\right)^{1+c} .
$$

If $\frac{k-1-l}{1-c}<k=\operatorname{dim}\left(\Omega_{2}\right)$, then the first term of the right hand of the inequality (3.5) is integrable. So,

$$
\int_{\Omega}\left|x_{2}\right|^{l}|u|^{b(1+c)} \leq B\left(\int_{\Omega}|u|^{b-1}|\nabla u|\right)^{1+c} .
$$


If $1<\frac{p}{p-1}(b-1)<p^{*}$, by the Hölder inequality and Sobolev embedding theorem, we get

$$
\int_{\Omega}\left|x_{2}\right|^{l}|u|^{b(1+c)} \leq B\left(\int_{\Omega}|\nabla u|^{p}\right)^{\frac{b(1+c)}{p}} .
$$

Now let $b=\frac{p k}{1+k-p}$ and $c=\frac{1+l^{\prime}-\theta}{k}$, where $\theta$ is small enough and $0<l^{\prime}<l$. By elementary calculation, $b$ and $c$ satisfy the following inequalities

$$
\left\{\begin{array}{l}
\frac{k-1-l}{1-c}<k \\
1<\frac{p}{p-1}(b-1)<p^{*}
\end{array}\right.
$$

Thus we complete the proof.

From the above discussion, we can give a more general result. Suppose $h(x)$ satisfy $\left(\mathbf{H}_{\mathbf{1}}\right)$ and the following condition:

$\left(\mathbf{H}_{2}\right): l_{h}>0$, where $l_{h}=\sup \left\{\lambda>0:|h(x)| /\left|x_{2}\right|^{\lambda}<\infty, x \in \Omega\right\}$.

Then we have the following theorem:

Theorem 3.4. Suppose that $h(x)$ satisfy $\left(\mathbf{H}_{\mathbf{1}}\right),\left(\mathbf{H}_{\mathbf{2}}\right)$, then there exists a positive number $\tau=\tau(h, p, m, k)$ such that the embedding $W_{0, s}^{1, p}(\Omega) \hookrightarrow L_{h}^{q}(\Omega)$ is compact for all $q \in\left(1, p^{*}+\tau\right)$.

\section{Compact embedding for variable exponent Sobolev space}

For any nonempty subset $U \subset \Omega$, we denote $|U|$ be the $N$-dimensional Lebesgue measure of $U$, and $|\cdot|_{p(x), U}:=|\cdot|_{L^{p(x)}(U)},|\cdot|_{h, p(x), U}:=|\cdot|_{L_{h}^{p(x)}(U)}$, $\|\cdot\|_{1, p(x), U}:=\|\cdot\|_{W^{1, p(x)}(U)}$. For simplicity, we denote $p_{+}=p_{+}(\Omega), p_{-}=p_{-}(\Omega)$.

Theorem 4.1. Suppose that $h(x)$ satisfy $\left(\mathbf{H}_{\mathbf{1}}\right),\left(\mathbf{H}_{\mathbf{2}}\right)$ and $p(x), q(x) \in C_{+}(\bar{\Omega}) \cap$ $S(\Omega), p(x)<N$. Then there exists a constant $\tau=\tau\left(h, p_{-}, m, k\right)$ and a continuous compact embedding $W_{0, s}^{1, p(x)}(\Omega) \hookrightarrow L_{h}^{q(x)}(\Omega)$ for $p(x)<q(x)<p^{*}(x)+\tau$, $\forall x \in \bar{\Omega}$.

Proof. For positive constant $\alpha<N$, denote $\alpha^{*}$, the conjugate number of $\alpha$, that is

$$
\alpha^{*}=\frac{N \alpha}{N-\alpha} .
$$

If we consider $\alpha^{*}$ as a function of $\alpha$, then $\alpha^{*}$ is increasing with respect to $\alpha$. Thus, we get

$$
\left(p^{*}\right)_{-}=\inf \left(\frac{N p(x)}{N-p(x)}\right)=\frac{N \inf p(x)}{N-\inf p(x)}=\frac{N p_{-}}{N-p_{-}}=\left(p_{-}\right)^{*}, \quad \forall x \in \bar{\Omega} .
$$

Denote $p_{-}^{*}:=\left(p^{*}\right)_{-}=\left(p_{-}\right)^{*}$. Clearly,

$$
\begin{aligned}
& q(x)<p_{-}^{*}+\tau \leq p^{*}(x)+\tau, \quad \forall x \in \bar{\Omega}, \\
& q(x) \leq q_{+}<p^{*}(x)+\tau, \quad \forall x \in \bar{\Omega}
\end{aligned}
$$

for $q(x)<p^{*}(x)+\tau, \forall x \in \bar{\Omega}$.

In the following, we separate our proof into two cases. 
Case 1: $q_{+}<p_{-}^{*}+\tau$.

Due to $p(x) \geq p_{-}, \forall x \in \bar{\Omega}$, Proposition 2.7 implies that there is a continuous embedding

$$
W^{1, p(x)}(\Omega) \hookrightarrow W^{1, p_{-}}(\Omega)
$$

and so there exists $C_{1}>0$ such that

$$
\|u\|_{1, p_{-}} \leq C_{1}\|u\|_{1, p(x)}, \quad \forall u \in W^{1, p(x)}(\Omega) .
$$

Clearly, for $u \in W_{0, s}^{1, p(x)}(\Omega)$, we still have

$$
\|u\|_{1, p_{-}} \leq C_{1}\|u\|_{1, p(x)} .
$$

Since $q_{+}<p_{-}^{*}+\tau$ with $\tau=\tau\left(h, p_{-}, m, k\right)$, we have continuous and compact embedding

$$
W_{0, s}^{1, p_{-}}(\Omega) \hookrightarrow L_{h}^{q_{+}}(\Omega)
$$

by the Sobolev embedding Theorem 3.4. Then there exists $C_{2}>0$ such that

$$
|u|_{h, q_{+}} \leq C_{2}\|u\|_{1, p_{-}} .
$$

We assert that there is a continuous embedding

$$
L_{h}^{q_{+}}(\Omega) \hookrightarrow L_{h}^{q(x)}(\Omega)
$$

for $q(x) \leq q_{+}, \forall x \in \bar{\Omega}$.

Indeed, let $u \in L_{h}^{q_{+}}(\Omega)$, we observe that,

$$
\begin{aligned}
\int_{\Omega} h(x)|u|^{q(x)} & =\int_{\{x \in \Omega:|u| \leq 1\}} h(x)|u|^{q(x)}+\int_{\{x \in \Omega:|u|>1\}} h(x)|u|^{q(x)} \\
& \leq \int_{\{x \in \Omega:|u| \leq 1\}} h(x)+\int_{\Omega} h(x)|u|^{q_{+}} \\
& \leq\left|\Omega_{0}\right| \cdot\|h\|_{\infty}+|u|_{h, q_{+}}^{q_{+}}
\end{aligned}
$$

where $\Omega_{0}=\{x \in \Omega:|u| \leq 1\}$.

Denote $S:=\left\{v \in L_{h}^{q_{+}}(\Omega):|v|_{h, q_{+}}=1\right\}$, we have for any $v \in S$ that

$$
\int_{\Omega} h(x)|v|^{q(x)} \leq\left|\Omega_{0}\right| \cdot\|h\|_{\infty}+1 .
$$

Let $C_{3}=\left|\Omega_{0}\right| \cdot\|h\|_{\infty}+1$, utilizing (4.4) we get that

$$
\begin{aligned}
\int_{\Omega} h(x)\left|\frac{v}{C_{3}}\right|^{q(x)} & \leq \int_{\Omega} h(x) \frac{|v|^{q(x)}}{C_{3}} \\
& =\frac{1}{C_{3}} \int_{\Omega} h(x)|v|^{q(x)} \\
& \leq 1 .
\end{aligned}
$$

From the definition of $|\cdot|_{h, q(x)}$ and (4.5), we obtain

$$
|v|_{h, q(x)} \leq C_{3} .
$$


Since

$$
\left|\frac{u}{|u|_{h, q_{+}}}\right|_{h, q_{+}}=1
$$

clearly we have

$$
\frac{u}{|u|_{h, q_{+}}} \in S
$$

Thus, it follows from (4.6) that

$$
\left|\frac{u}{|u|_{h, q_{+}}}\right|_{h, q(x)} \leq C_{3},
$$

that is

$$
\int_{\Omega} h(x)\left|\frac{\frac{u}{|u|_{h, q_{+}}}}{C_{3}}\right|^{q(x)} \leq 1,
$$

and so

$$
\int_{\Omega} h(x)\left|\frac{u}{C_{3}|u|_{h, q_{+}}}\right|^{q(x)} d x \leq 1 .
$$

(4.7) implies that

$$
|u|_{h, q(x)} \leq C_{3}|u|_{h, q_{+}} .
$$

We have, as desired, obtained the continuous embedding $L_{h}^{q_{+}}(\Omega) \hookrightarrow L_{h}^{q(x)}(\Omega)$.

Consequently, combining (4.1), (4.2) and (4.8), we discover that $|u|_{h . q(x)} \leq$ $C\|u\|_{1, p(x)}$ for any $u \in W_{0, s}^{1, p(x)}(\Omega)$ and so the embedding $W_{0, s}^{1, p(x)}(\Omega) \hookrightarrow$ $L_{h}^{q(x)}(\Omega)$ is continuous. Its compactness is obtained by the compact embedding $W_{0, s}^{1, p_{-}}(\Omega) \hookrightarrow L_{h}^{q+}(\Omega)$.

Case 2: $q_{+}<p_{-}^{*}+\tau$ does not hold.

In this case, we can find a finite number of subsets $\Omega_{i j} \subset \Omega=\Omega_{1} \times \Omega_{2}$, $i=1, \ldots, s, j=1, \ldots, t$ such that $\bar{\Omega}=\cup_{i=1}^{t} \cup_{j=1}^{s} \bar{\Omega}_{i j}$ and on every $\Omega_{i j}$ there holds

$$
q_{+}\left(\Omega_{i j}\right)<p_{-}^{*}\left(\Omega_{i j}\right)+\tau .
$$

Precisely, we first decompose $\Omega_{1} \subset \mathbb{R}^{m}$. Divide $\mathbb{R}^{m}$ into the disjoint open $m$-cubes $\left\{I_{i}, i=1,2, \ldots\right\}$ with side $l>0$ such that $\mathbb{R}^{m}=\cup_{i=1}^{\infty} \bar{I}_{i}$. Let $\Omega_{1 i}=\Omega_{1} \cap I_{i}$, since $\Omega_{1}$ is bounded, there exists a positive integer $s$ such that $\Omega_{1 i}=\emptyset$ for any $i>s$. That is, we have $\bar{\Omega}_{1}=\cup_{i=1}^{s} \bar{\Omega}_{1 i}$.

Next, we divide $\Omega_{2}=B(0, R) \subset \mathbb{R}^{k}$ to $\left\{\Omega_{2 j}\right\}_{j=1}^{t}$ such that for $r=$ $\frac{R}{t}, \Omega_{21}=B(0, r), \Omega_{22}=B(0,2 r) \backslash B(0, r), \ldots, \Omega_{2 t}=B(0, R) \backslash B(0,(t-1) r)$. Hence, $\bar{\Omega}_{2}=\cup_{j=1}^{t} \bar{\Omega}_{2 j}$.

Clearly, $\Omega$ has been divided into a finite number of subsets $\Omega_{i j}=\Omega_{1 i} \times$ $\Omega_{2 j}, i=1, \ldots, s, j=1, \ldots, t$ such that $\bar{\Omega}=\cup_{i=1}^{s} \cup_{j=1}^{t} \bar{\Omega}_{i j}$.

Because $p(x), q(x)$ are radically symmetric about $x_{2} \in \Omega_{2}$, we get that $p\left(x_{1}, x_{2}\right)=p\left(x_{1},\left|x_{2}\right|\right)$ and $q\left(x_{1}, x_{2}\right)=q\left(x_{1},\left|x_{2}\right|\right)$. Then, choose $r, l>0$ small 
enough, utilizing the continuity of $p(x), q(x)$ and the assumption $p(x)<q(x)<$ $p^{*}(x)+\tau$, we deduce that

$$
p_{+}\left(\Omega_{i j}\right)<q_{-}\left(\Omega_{i j}\right) \leq q_{+}\left(\Omega_{i j}\right)<p_{-}^{*}\left(\Omega_{i j}\right)+\tau
$$

for any given $i, j$.

Now, for any $u \in W_{0, s}^{1, p(x)}(\Omega)$, we will show that $u \in L_{h}^{q(x)}(\Omega)$. It is obvious that if $u \in W_{0, s}^{1, p(x)}(\Omega)$ then $u \in W_{s}^{1, p(x)}\left(\Omega_{i j}\right)$ for $\forall i=1, \ldots, s, j=1, \ldots, t$ and $u=0$ on $\partial \Omega$. Let $l, r>0$ be chosen small enough such that

$$
\int_{\Omega_{i j}}|u|^{p(x)}+|\nabla u|^{p(x)} \leq 1, \quad \forall i=1, \ldots, s, \quad j=1, \ldots, t .
$$

If $|u|_{h, q(x), \Omega_{i j}} \leq 1$, it easily follows from Remark 2.3 that

$$
\int_{\Omega_{i j}} h(x)|u|^{q(x)} \leq|u|_{h, q(x), \Omega_{i j}}^{q-\left(\Omega_{i j}\right)} .
$$

By (4.3), we have $L_{h}^{q+\left(\Omega_{i j}\right)}\left(\Omega_{i j}\right) \hookrightarrow L_{h}^{q(x)}\left(\Omega_{i j}\right)$, and so

$$
|u|_{h, q(x), \Omega_{i j}} \leq C_{3}|u|_{h, q_{+}, \Omega_{i j}} .
$$

Since $q_{+}\left(\Omega_{i j}\right)<p_{-}^{*}\left(\Omega_{i j}\right)+\tau=\left(p_{-}\left(\Omega_{i j}\right)\right)^{*}+\tau$, by (4.2) we get that

$$
|u|_{h, q_{+}\left(\Omega_{i j}\right), \Omega_{i j}} \leq C_{2}\|u\|_{1, p_{-}\left(\Omega_{i j}\right), \Omega_{i j}}, \quad \forall i=1, \ldots, s, j=1, \ldots t .
$$

From (4.1), we see that

$$
\|u\|_{1, p_{-}\left(\Omega_{i j}\right), \Omega_{i j}} \leq C_{1}\|u\|_{1, p(x), \Omega_{i j}} \text { for } u \in W_{s}^{1, p(x)}\left(\Omega_{i j}\right), \quad \forall i=1, \ldots, s, j=1, \ldots t .
$$

Moreover, $C_{1}, C_{2}, C_{3}$ in the above three inequalities are independent of $i, j$.

Therefore, we obtain that

$$
\begin{aligned}
\int_{\Omega_{i j}} h(x)|u|^{q(x)} & \leq|u|_{h, q(x), \Omega_{i j}}^{q-\left(\Omega_{i j}\right)} \\
& \leq\left(C_{3}|u|_{h, q_{+}\left(\Omega_{i j}\right), \Omega_{i j}}\right)^{q_{-}\left(\Omega_{i j}\right)} \\
& \leq\left(C_{2} C_{3}\|u\|_{1, p_{-}\left(\Omega_{i j}\right), \Omega_{i j}}\right)^{q_{-}\left(\Omega_{i j}\right)} \\
& \leq\left(C_{1} C_{2} C_{3}\|u\|_{1, p(x), \Omega_{i j}}\right)^{q_{-}\left(\Omega_{i j}\right)} \\
& =\left(C_{1} C_{2} C_{3}\right)^{q_{-}\left(\Omega_{i j}\right)}\|u\|_{1, p(x), \Omega_{i j}}^{q-\left(\Omega_{i j}\right)} \\
& =C_{4}\|u\|_{1, p(x), \Omega_{i j}}^{q} .
\end{aligned}
$$

Utilizing Remak 2.4, we have

$$
c_{3}\|u\|_{1, p(x), \Omega_{i j}}^{p_{+}\left(\Omega_{i j}\right)} \leq \int_{\Omega_{i j}}|u|^{p(x)}+|\nabla u|^{p(x)} .
$$


It follows that

$$
\begin{aligned}
\int_{\Omega_{i j}} h(x)|u|^{q(x)} & \leq \frac{C_{4}}{c_{3}}\left(\int_{\Omega_{i j}}|u|^{p(x)}+|\nabla u|^{p(x)}\right)^{\frac{q_{-}\left(\Omega_{i j}\right)}{p_{+}\left(\Omega_{i j}\right)}} \\
& =C\left(\int_{\Omega_{i j}}|u|^{p(x)}+|\nabla u|^{p(x)}\right)^{\frac{q_{-}\left(\Omega_{i j}\right)}{p_{+}\left(\Omega_{i j}\right)}} .
\end{aligned}
$$

Since $p_{+}\left(\Omega_{i j}\right)<q_{-}\left(\Omega_{i j}\right)$ for any given $i, j$, we have

$$
\left(\int_{\Omega_{i j}}|u|^{p(x)}+|\nabla u|^{p(x)}\right)^{\frac{q_{-}\left(\Omega_{i j}\right)}{p+\left(\Omega_{i j}\right)}} \leq \int_{\Omega_{i j}}|u|^{p(x)}+|\nabla u|^{p(x)} .
$$

Then, it is easy to see that

$$
\int_{\Omega_{i j}} h(x)|u|^{q(x)} \leq C \int_{\Omega_{i j}}|u|^{p(x)}+|\nabla u|^{p(x)}
$$

for $C$ independent of $i, j$.

If $|u|_{h, q(x), \Omega_{i j}} \geq 1$, similarly we get

$$
\int_{\Omega_{i j}} h(x)|u|^{q(x)} \leq C \int_{\Omega_{i j}}|u|^{p(x)}+|\nabla u|^{p(x)} .
$$

Consequently,

$$
\begin{aligned}
\int_{\Omega} h(x)|u|^{q(x)} & =\int_{\cup_{i=1}^{s} \cup_{j=1}^{t} \Omega_{i j}} h(x)|u|^{q(x)} \\
& =\sum_{i=1}^{s} \sum_{j=1}^{t} \int_{\Omega_{i j}} h(x)|u|^{q(x)} \\
& \leq \sum_{i=1}^{s} \sum_{j=1}^{t} C\left(\int_{\Omega_{i j}}|u|^{p(x)}+|\nabla u|^{p(x)}\right) \\
& =C \sum_{i=1}^{s} \sum_{j=1}^{t}\left(\int_{\Omega_{i j}}|u|^{p(x)}+|\nabla u|^{p(x)}\right) \\
& =C\left(\int_{\Omega}|u|^{p(x)}+|\nabla u|^{p(x)}\right) .
\end{aligned}
$$

We have, as desired, proved that the embedding $W_{0, s}^{1, p(x)}(\Omega) \hookrightarrow L_{h}^{q(x)}(\Omega)$ is continuous. The compactness of the embedding follows easily from X. L. Fan (see [15], proof of Theorem 1.3).

\section{An application}

As in the previous sections, let $\Omega=\Omega_{1} \times \Omega_{2} \subset \mathbb{R}^{N}$, with $\Omega_{1} \subset \mathbb{R}^{m}(m \geq 1)$ being a bounded regular domain, and $\Omega_{2} \subset \mathbb{R}^{k}(k \geq 2)$ being a ball of radius $\mathrm{R}$, centered at the origin. 
Now we consider the problem

$$
\left\{\begin{array}{l}
-\Delta_{p(x)} u \equiv-\operatorname{div}\left(|\nabla u|^{p(x)-2} \nabla u\right)=\lambda f_{1}(x, u)+h(x) f_{2}(x, u) \quad x \in \Omega, \\
u=0 \quad x \in \partial \Omega
\end{array}\right.
$$

where $\lambda$ is a positive real parameter, $p(x) \in C_{+}(\bar{\Omega}) \cap S(\Omega), h(x)$ satisfy $\left(\mathbf{H}_{\mathbf{1}}\right)$, $\left(\mathbf{H}_{2}\right)$.

Suppose that $f_{i}: \Omega \times \mathbb{R} \rightarrow \mathbb{R}, i=1,2$ satisfies Caratheodory condition and the following assumptions:

$\left(\mathbf{O}_{1}\right):\left|f_{i}(x, t)\right| \leq c_{1}+c_{2}|t|^{q_{i}(x)-1}, \forall(x, t) \in \bar{\Omega} \times \mathbb{R}, i=1,2$, where $q_{1}(x) \in$ $C_{+}(\bar{\Omega}), q_{2}(x) \in C_{+}(\bar{\Omega}) \cap S(\Omega), q_{1}(x)<p(x)<q_{2}(x)<p^{*}(x)+\tau$, $\forall x \in \bar{\Omega}$, and $\left(q_{1}\right)_{+}<p_{-}, p_{+}<\left(q_{2}\right)_{-}$.

$\left(\mathbf{O}_{2}\right): \exists M>0, \theta \in\left(0, \frac{1}{p_{+}}\right)$such that $0<F_{2}(x, t) \leq \theta t f_{2}(x, t)$ for $|t| \geq M$, $\forall x \in \Omega$, where $F_{2}(x, t)=\int_{0}^{t} f_{2}(x, s) d s$.

$\left(\mathbf{O}_{3}\right): \exists \delta>0, c_{3}>0$ and $q_{3}(x) \in C(\bar{\Omega})$ with $\left(q_{3}\right)_{+}<p_{-}$such that

$$
F_{1}(x, t) \geq c_{3}|t|^{q_{3}(x)}, \quad \forall x \in \Omega, \quad \forall|t| \leq \delta,
$$

where $F_{1}(x, t)=\int_{0}^{t} f_{1}(x, s) d s$.

Remark 5.1. From $\left(\mathbf{O}_{1}\right)$ and $\left(\mathbf{O}_{3}\right)$, we get $F_{2}(x, t) \geq c_{4}|t|^{\frac{1}{\theta}}-c_{5}, \forall(x, t) \in \bar{\Omega} \times \mathbb{R}$.

Definition 5.2. We call that $u \in W_{0}^{1, p(x)}(\Omega)$ is a weak solution of $(\mathrm{P})$, if $\int_{\Omega}|\nabla u|^{p(x)-2} \nabla u \nabla v d x=\int_{\Omega} \lambda f_{1}(x, u) v d x+\int_{\Omega} h(x) f_{2}(x, u) v d x, \forall v \in W_{0}^{1, p(x)}(\Omega)$.

We denote $\|u\|_{p(x)}=|\nabla u|_{p(x)}$ be the equivalent norm to $\|u\|_{1, p(x)}$ for $u \in W_{0}^{1, p(x)}(\Omega)$. According to Theorem 4.1, the embedding mapping $i: W_{0, s}^{1, p(x)}(\Omega) \rightarrow L_{h}^{q(x)}(\Omega)$ is compact. Then we can define the functional $I: W_{0, s}^{1, p(x)}(\Omega) \hookrightarrow \mathbb{R}$ by

$$
I(u)=\int_{\Omega} \frac{1}{p(x)}|\nabla u|^{p(x)} d x-\int_{\Omega} \lambda F_{1}(x, u) d x-\int_{\Omega} h(x) F_{2}(x, u) d x .
$$

Obviously, $I$ is well-defined in $W_{0, s}^{1, p(x)}(\Omega)$ and is of class $C^{1}$. Therefore, the weak solutions of $(\mathrm{P})$ are exactly the critical points of $I$. In the following, we give two propositions which are useful in our later proof.

Proposition 5.3 (see [9]). Let $J(u)=\int_{\Omega} \frac{1}{p(x)}|\nabla u|^{p(x)} d x$ for $u \in W_{0}^{1, p(x)}(\Omega)$; then $J \in C^{1}\left(W_{0}^{1, p(x)}(\Omega), \mathbb{R}\right)$ and the $p(x)$-Laplacian is the derivative operator of $J$. We define $J^{\prime}: W_{0}^{1, p(x)}(\Omega) \rightarrow\left(W_{0}^{1, p(x)}(\Omega)\right)^{*}$; then

$$
\left(J^{\prime}(u), v\right)=\int_{\Omega}|\nabla u|^{p(x)-2} \nabla u \nabla v d x, \quad \forall u, v \in W_{0}^{1, p(x)}(\Omega),
$$

and $J^{\prime}$ satisfies the following properties:

(i) $J^{\prime}$ is a continuous, bounded, strictly monotone operator, and is a homeomorphism.

(ii) $J^{\prime}$ is a mapping of type $\left(S_{+}\right)$, i.e., if $u_{n} \rightarrow u$ in $W_{0}^{1, p(x)}(\Omega)$ and $\lim _{n \rightarrow \infty}\left(J^{\prime}\left(u_{n}\right)-J^{\prime}(u), u_{n}-u\right) \leq 0$, then $u_{n} \rightarrow u$ in $W_{0}^{1, p(x)}(\Omega)$. 
Proposition 5.4 (see [6]). Let $\Phi_{1}=\int_{\Omega} \lambda F_{1}(x, u) d x, \Phi_{2}=\int_{\Omega} h(x) F_{2}(x, u) d x$, then for $i=1,2, \Phi_{i}$ and $\Phi_{i}^{\prime}$ are weakly-strongly continuous, i.e., $u_{n} \rightarrow u$ implies $\Phi_{i}\left(u_{n}\right) \rightarrow \Phi_{i}(u)$ and $\Phi_{i}^{\prime}\left(u_{n}\right) \rightarrow \Phi_{i}^{\prime}(u)$. Additionally, $I^{\prime}=J^{\prime}-\Phi_{1}^{\prime}-\Phi_{2}^{\prime}$ is also of type $\left(S_{+}\right)$.

Theorem 5.5. Assume $\left(\mathbf{O}_{1}\right),\left(\mathbf{O}_{2}\right),\left(\mathbf{O}_{3}\right)$ hold, then there exists $\lambda_{0}>0$ such that for $\forall \lambda \in\left(0, \lambda_{0}\right)$, the problem $(P)$ has two weak solutions $u_{1}, u_{2}$ with $I\left(u_{1}\right)>0$ and $I\left(u_{2}\right)<0$.

Proof. First, we show that $I$ satisfies the $(P S)$ condition, i.e., any sequence $\left\{u_{n}\right\}$ in $W_{0, s}^{1, p(x)}(\Omega)$ such that $I\left(u_{n}\right) \rightarrow c$ and $I^{\prime}\left(u_{n}\right) \rightarrow 0$ has a convergence subsequence. For any $(P S)$ sequence, we have

$$
\begin{aligned}
I\left(u_{n}\right)-\theta I^{\prime}\left(u_{n}\right) u_{n} & =c-\theta I^{\prime}\left(u_{n}\right) u_{n} \\
& \leq c+\theta\left|I^{\prime}\left(u_{n}\right) u_{n}\right| \\
& \leq C+C\left\|u_{n}\right\|_{p(x)} .
\end{aligned}
$$

Moreover,

$$
\begin{aligned}
I\left(u_{n}\right)-\theta I^{\prime}\left(u_{n}\right) u_{n}= & \int_{\Omega} \frac{1}{p(x)}\left|\nabla u_{n}\right|^{p(x)} d x-\int_{\Omega} \lambda F_{1}\left(x, u_{n}\right) d x \\
& -\int_{\Omega} h(x) F_{2}\left(x, u_{n}\right) d x \\
& -\theta\left(\int_{\Omega}\left|\nabla u_{n}\right|^{p(x)} d x-\int_{\Omega} \lambda f_{1}\left(x, u_{n}\right) u_{n} d x\right. \\
& \left.-\int_{\Omega} h(x) f_{2}\left(x, u_{n}\right) u_{n} d x\right) \\
= & \int_{\Omega}\left(\frac{1}{p(x)}-\theta\right)\left|\nabla u_{n}\right|^{p(x)} d x \\
& -\int_{\Omega} \lambda\left(F_{1}\left(x, u_{n}\right)-f_{1}\left(x, u_{n}\right) u_{n}\right) d x \\
& -\int_{\Omega} h(x)\left(F_{2}\left(x, u_{n}\right)-f_{2}\left(x, u_{n}\right) u_{n}\right) d x \\
\geq & \left(\frac{1}{p_{+}}-\theta\right)\left\|u_{n}\right\|_{p(x)}^{p_{-}} \\
& -\int_{\Omega} \lambda\left(F_{1}\left(x, u_{n}\right)-f_{1}\left(x, u_{n}\right) u_{n}\right) d x \\
& -\int_{\Omega} h(x)\left(F_{2}\left(x, u_{n}\right)-f_{2}\left(x, u_{n}\right) u_{n}\right) d x . \\
&
\end{aligned}
$$

By $\left(\mathbf{O}_{1}\right)$, we get

$$
\begin{aligned}
F_{1}(x, t)-\theta f_{1}(x, t) t & \leq\left|F_{1}(x, t)\right|+\theta\left|f_{1}(x, t)\right||t| \\
& \leq \int_{0}^{t}\left(c_{1}+c_{2}|s|^{q_{1}(x)-1}\right) d s+\theta\left(c_{1}+c_{2}|t|^{q_{1}(x)-1}\right)|t| \\
& \leq C_{1}+C_{2}|t|^{q_{1}(x)} .
\end{aligned}
$$


$\left(\mathbf{O}_{3}\right)$ implies that

$$
F_{2}(x, t)-\theta t f_{2}(x, t) \leq\left(\theta t f_{2}(x, t)+C_{3}\right)-\theta t f_{2}(x, t)=C_{3} .
$$

Combining the above inequalities (5.1)-(5.4), we obtain

$$
\begin{aligned}
C+C\left\|u_{n}\right\|_{p(x)} \geq & \left(\frac{1}{p_{+}}-\theta\right)\left\|u_{n}\right\|_{p(x)}^{p_{-}}-\int_{\Omega}\left(C_{1}+C_{2}\left|u_{n}\right|^{q_{1}(x)}\right) d x \\
& -\int_{\Omega} h(x) C_{3} d x \\
\geq & \left(\frac{1}{p_{+}}-\theta\right)\left\|u_{n}\right\|_{p(x)}^{p_{-}}-C_{4} \int_{\Omega}\left|u_{n}\right|^{q_{1}(x)} d x-C_{5} \\
\geq & \left(\frac{1}{p_{+}}-\theta\right)\left\|u_{n}\right\|_{p(x)}^{p_{-}}-C_{4}\left|u_{n}\right|_{q_{1}(x)}^{\left(q_{1}\right)_{+}} d x-C_{5} \\
\geq & \left(\frac{1}{p_{+}}-\theta\right)\left\|u_{n}\right\|_{p(x)}^{p_{-}}-C_{6}\left\|u_{n}\right\|_{p(x)}^{\left(q_{1}\right)_{+}} d x-C_{5} .
\end{aligned}
$$

It follows from $1<\left(q_{1}\right)_{+}<p_{-}$that $\left\{u_{n}\right\}$ is bounded.

Since $W_{0}^{1, p(x)}(\Omega)$ is a reflexive Banach space, we can find a weakly convergence subsequence $\left\{u_{n_{i}}\right\} \subset W_{0}^{1, p(x)}(\Omega)$. For $u_{n_{i}} \rightarrow u$ and $I^{\prime}\left(u_{n_{i}}\right) \rightarrow 0$, we see from Proposition 5.4 that $u_{n_{i}} \rightarrow u$. Then we select $\left\{u_{n_{j}}\right\} \subset\left\{u_{n_{i}}\right\}$ such that $u_{n_{j}} \in S(\Omega)$ for any $j$, and still we have $u_{n_{j}} \rightarrow u$ in $W_{0, s}^{1, p(x)}(\Omega)$. This shows that $I$ satisfies $(P S)$ condition.

Next, for sufficiently small $\|u\|_{p(x)}$, we get

$$
\begin{aligned}
\Phi_{2}(u) & =\int_{\Omega} h(x) F_{2}(x, u) d x \\
& \leq \int_{\Omega} h(x)\left(c_{1}+c_{2}|u|^{q_{2}(x)}\right) d x \\
& \leq C+c_{2} \int_{\Omega} h(x)|u|^{q_{2}(x)} d x \\
& \leq C+c_{2}|u|_{h(x), q_{2}(x)}^{\left(q_{2}\right)_{-}} \\
& \leq C+C\|u\|_{p(x)}^{\left(q_{2}\right)_{-}} .
\end{aligned}
$$

Thus,

$$
J(u)-\Phi_{2}(u) \geq \frac{1}{p_{+}}\|u\|_{p(x)}^{p_{+}}-C\|u\|_{p(x)}^{\left(q_{2}\right)_{-}}-C .
$$

Since $p_{+}<\left(q_{2}\right)_{-}$, there exists $\gamma>0$ and $\alpha>0$ such that $J(u)-\Phi_{2}(u) \geq \alpha$ for $\|u\|=\gamma$. 
In light of $\left(\mathbf{O}_{1}\right)$,

$$
\begin{aligned}
\Phi_{1}(u) & =\lambda \int_{\Omega} F_{1}(x, u) d x \\
& \leq \lambda \int_{\Omega}\left(c_{1}+c_{2}|u|^{q_{1}(x)}\right) d x \\
& \leq \lambda\left(C+c_{2}|u|_{q_{1}(x)}^{\left(q_{1}\right)_{-}}\right) \\
& \leq \lambda\left(C+C\|u\|_{p(x)}^{\left(q_{1}\right)_{-}}\right) .
\end{aligned}
$$

Clearly, We can find $\lambda_{0}>0$ such that for any $\lambda \in\left(0, \lambda_{0}\right)$, we have $\Phi_{1}(u) \leq \frac{\alpha}{2}$ for $\|u\|=\gamma$. So when $\lambda \in\left(0, \lambda_{0}\right)$, we obtain

$$
I(u)=J(u)-\Phi_{1}(u)-\Phi_{2}(u) \geq \frac{\alpha}{2}>0 \quad \text { for } \quad\|u\|=\gamma .
$$

Furthermore,

$$
\begin{aligned}
I(u) & =\int_{\Omega} \frac{1}{p(x)}|\nabla u|^{p(x)} d x-\int_{\Omega} \lambda F_{1}(x, u) d x-\int_{\Omega} h(x) F_{2}(x, u) d x \\
& \leq \frac{1}{p_{-}}\|u\|^{p_{+}}+\lambda \int_{\Omega}\left(c_{1}+c_{2}|u|^{q_{1}(x)}\right) d x-\int_{\Omega} h(x)\left(c_{4}|u|^{\frac{1}{\theta}}-c_{5}\right) d x \\
& \leq \frac{1}{p_{-}}\|u\|^{p_{+}}+\lambda C|u|_{q_{1}(x)}^{\left(q_{1}\right)_{+}}-c_{4} \int_{\Omega} h(x)|u|^{\frac{1}{\theta}} d x-C \\
& \leq \frac{1}{p_{-}}\|u\|^{p_{+}}+\lambda C\|u\|_{p(x)}^{\left(q_{1}\right)_{+}}-C|u|_{h(x), \frac{1}{\theta}}^{\frac{1}{\theta}}-C \\
& \leq \frac{1}{p_{-}}\|u\|^{p_{+}}+\lambda C\|u\|_{p(x)}^{\left(q_{1}\right)_{+}}-C\|u\|_{p(x)}^{\frac{1}{\theta}} d x-C .
\end{aligned}
$$

The assumption $\left(q_{1}\right)_{+}<p_{+}<\frac{1}{\theta}$ suffices to get that there exists a $\omega \in$ $W_{0, s}^{1, p(x)}(\Omega)$ such that

$$
I(\omega)<0 \text { for }\|\omega\|_{p(x)}>\gamma .
$$

Noting that $I(0)=0$, combining (5.5) and (5.6) we obtain from Mountain Pass Theorem that $(P)$ has a nontrivial weak solution $u_{1}$ such that $I\left(u_{1}\right)>0$ for $\lambda \in\left(0, \lambda_{0}\right)$.

Now, taking $v_{0} \in C_{0}^{\infty}(\Omega)$ such that $0<\left|v_{0}\right| \leq \delta$. For $\alpha \in(0,1)$ small enough, according to $\left(\mathbf{O}_{\mathbf{1}}\right),\left(\mathbf{O}_{\mathbf{3}}\right)$ and Remark 5.1, we get

$$
\begin{aligned}
I\left(\alpha v_{0}\right)= & \int_{\Omega} \frac{1}{p(x)}\left|\alpha v_{0}\right|^{p(x)} d x-\lambda \int_{\Omega} F_{1}\left(x, \alpha v_{0}\right) d x-\int_{\Omega} h(x) F_{2}\left(x, \alpha v_{0}\right) d x \\
\leq & \alpha^{p_{-}} \int_{\Omega} \frac{1}{p_{-}}\left|v_{0}\right|^{p(x)} d x-\lambda \int_{\Omega} c_{3}\left|\alpha v_{0}\right|^{q_{3}(x)} d x \\
& +\int_{\Omega} h(x)\left(c_{1}+c_{2}\left|\alpha v_{0}\right|^{q_{2}(x)}\right) d x \\
\leq & C \alpha^{p_{-}} \int_{\Omega}\left|v_{0}\right|^{p(x)} d x-\lambda c_{3} \alpha^{\left(q_{3}\right)}+\int_{\Omega}\left|v_{0}\right|^{q_{3}(x)} d x \\
& +C \alpha^{\left(q_{2}\right)_{-}} \int_{\Omega} h(x)\left|v_{0}\right|^{q_{2}(x)} d x+C .
\end{aligned}
$$


Clearly, $\left(q_{3}\right)_{+}<p_{-}<\left(q_{2}\right)_{-}$yields that $I\left(\alpha v_{0}\right)<0$ for $\alpha \in(0,1)$ small enough. Now, choose $\alpha_{0}$ so small that $\left\|\alpha_{0} v_{0}\right\|_{p(x)}<\gamma$ and $I\left(\alpha_{0} v_{0}\right)<0$.

Set

$$
\mathcal{A}:=\left\{u \in W_{0, s}^{1, p(x)}(\Omega):\|u\|_{p(x)} \leq \gamma\right\},
$$

then we have $\beta:=\min _{u \in \mathcal{A}} I(u)<0$. We claim that this minimum is achieved at some $u_{1}$. To see this, we select a minimizing sequence $\left\{u_{n}\right\} \subset \mathcal{A} \subset W_{0, s}^{1, p(x)}(\Omega)$, then $I\left(u_{n}\right) \rightarrow \beta$. have

Because $\mathcal{A}$ is bounded, we get $u_{n} \rightarrow u_{2}$ in $\mathcal{A}$. Using Fatou Lemma, we

$$
\int_{\Omega} \frac{1}{p(x)}\left|\nabla u_{2}\right|^{p(x)} d x \leq \liminf _{n \rightarrow \infty} \int_{\Omega} \frac{1}{p(x)}\left|\nabla u_{n}\right|^{p(x)} d x .
$$

Moreover, we follow from Theorem 4.1 that

$$
\Phi_{i}\left(u_{n}\right) \rightarrow \Phi_{i}\left(u_{2}\right) \quad \text { for } i=1,2 .
$$

Consequently,

$$
\begin{aligned}
I\left(u_{2}\right) & =\int_{\Omega} \frac{1}{p(x)}\left|\nabla u_{2}\right|^{p(x)} d x-\Phi_{1}\left(u_{2}\right)-\Phi_{2}\left(u_{2}\right) \\
& \leq \liminf _{n \rightarrow \infty} \int_{\Omega} \frac{1}{p(x)}\left|\nabla u_{n}\right|^{p(x)} d x-\lim _{n \rightarrow \infty} \Phi_{1}\left(u_{n}\right)-\lim _{n \rightarrow \infty} \Phi_{2}\left(u_{n}\right) \\
& =\liminf _{n \rightarrow \infty} I\left(u_{n}\right) \\
& =\beta .
\end{aligned}
$$

Since $u_{2} \in \mathcal{A}$, then

$$
I\left(u_{2}\right) \geq \beta
$$

From (5.7) and (5.8) we discover that $I\left(u_{2}\right)=\beta$. That is, $u_{2}$ is a local minimizer of $I$ in $\mathcal{A}$ with $I\left(u_{2}\right)<0$. Our proof is complete.

\section{Acknowledgment}

The authors thanks the referee for useful comments and suggestions.

\section{References}

[1] Lions, P.L.: Symetrie et compacite dans les espaces de Sobolev. J. Funct. Anal. 49, 315-334 (1982)

[2] Lions, P.L.: The concentration-compactness principle in the calculus of variations, the locally compact case. Ann. Inn. H. Poincare Anal. Non Lineaire 1, 109-145 (1984)

[3] Adams, R.A., Fournier, J.F.: Sobolev Spaces. Academic Press, Dublin (2003)

[4] Wang, W.Z.: Sobolev embeddings involving symmetry. Bull. Sci. Math. 130, 269-278 (2006) 
[5] Marcellini, P.: Regularity and existence of solutions of elliptic equations with (p,q)-growth conditions. J. Differ. Equ. 90, 1-30 (1991)

[6] Fan, X.L.: Solutions for $p(x)$-Laplacian Dirichlet problems with singular coefficients. J. Math. Anal. Appl. 312, 464-477 (2005)

[7] Fan, X.L.: Some results on variable exponent analysis. In: Proceedings of 5th ISAAC Congress, University of Catania, Italy, July 25-30 (2005)

[8] Fan, X.L., Zhang, Q.H.: Existence of solutions for $p(x)$-Laplacian Dirichlet problem. Non. Anal. 52, 1843-1952 (2003)

[9] Fan, X.L., Deng, S.G.: Remarks on Ricceri's variational principle and applications to the $p(x)$-Laplacian equations. Non. Anal. 67(11), 3064-3075 (2007)

[10] Cruz-Uribe, D., Fiorenza, A., Martell, J.M., Pérez, C.: The boundedness of classical operators on variable $L^{p}$ spaces. Ann. Acad. Sci. Fenn. Math. 31, 239264 (2006)

[11] Edmunds, D.E., Rákosník, J.: Sobolev embedding with variable exponent. Studia Math. 143, 267-293 (2000)

[12] Edmunds, D.E., Rákosník, J.: Sobolev embedding with variable exponent, II. Math. Nachr. 246(247), 53-67 (2002)

[13] Kováčik, O., Rákosník, J.: On spaces $L^{p(x)}(\Omega)$ and $W^{k, p(x)}(\Omega)$. Czechoslovak Math. J. 41(116), 592-618 (1991)

[14] Fan, X.L., Zhao, D.: On the spaces $L^{p(x)}(\Omega)$ and $W^{m, p(x)}(\Omega)$. J. Math. Anal. Appl. 263, 424-446 (2001)

[15] Fan, X.L., Shen, J.S., Zhao, D.: Sobolev embedding theorems for spaces $W^{k, p(x)}(\Omega)$. J. Math. Anal. Appl. 262, 749-760 (2001)

[16] Fan, X.L., Zhao, Y.Z., Zhao, D.: Compact imbedding theorems with symmetry of Strauss-Lions type for the space $W^{1, p(x)}(\Omega)$. J. Math. Anal. Appl. 255, 333-348 (2001)

[17] Yao, J.H., Wang, X.Y.: On an open problem involving the $p(x)$-Laplacian: a further study on the multiplicity of weak solutions to $p(x)$-Laplacian equations. Non. Anal. 69, 1445-1453 (2008)

[18] Gilbarg, D., Trudinger, N.S.: Elliptic Partial Differential Equations of Second Order, 2nd edn. Spinger, Berlin (1983)

J. Gao, P. Zhao

School of Mathematics and Statistics,

Lanzhou University,

730000 Lanzhou,

People's Republic of China

e-mail: gaojj03@gmail.com 
P. Zhao

e-mail: zhaoph@lzu.edu.cn

Y. Zhang

Department of Mathematics and Computer Science, Chizhou University,

247000 Chizhou,

People's Republic of China

e-mail: zhangyong-hn@163.com

Received: 16 April 2009.

Accepted: 30 October 2009. 